\section{The first reported case of Bartonella endocarditis in Thailand}

\author{
Orathai Pachirat, ${ }^{1}$ Michael Kosoy, ${ }^{2}$ \\ Ying Bai, ${ }^{2}$ Sompop Prathani, ${ }^{1}$ \\ Anucha Puapairoj, ${ }^{1}$ Nordin Zeidner, \\ Leonard F. Peruski, ${ }^{3}$ Henry Baggett, ${ }^{3}$ \\ George Watt, ${ }^{3}$ Susan A. Maloney ${ }^{3}$ \\ ${ }^{1}$ Faculty of Medicine, Khon Kaen \\ University, Khon Kaen, Thailand; \\ ${ }^{2}$ Bacterial Diseases Branch, Division \\ of Vector Borne Infectious Diseases, \\ Centers for Disease Control and \\ Prevention, Fort Collins, Colorado USA; \\ ${ }^{3}$ International Emerging Infections \\ Program (IEIP), Thailand MOPH-US CDC \\ Collaboration, GDD RegionalCenter, US \\ CDC SE Asia Regional Office, \\ Nonthaburi, Thailand
}

\section{Abstract \\ Bartonella species have been shown to cause acute, undifferentiated fever in Thailand. A study to identify causes of endo- carditis that were blood culture-negative using routine methods led to the first reported case in Thailand of Bartonella endocarditis A 57 year-old male with underlying rheumatic heart disease presented with severe congestive heart failure and suspected infective endo- carditis. The patient underwent aortic and mitral valve replacement. Routine hospital blood cultures were negative but $B$. henselae was identified by serology, PCR, immunohisto- chemistry and specific culture techniques.}

\section{Introduction}

Blood culture-negative endocarditis (BCNE) is a term used in the cardiology literature to describe cases of infective endocarditis for which there is no bacterial growth in three independent blood samples cultured on standard aerobic media after seven days of incubation and subculturing. ${ }^{1}$ However, with specialized culture techniques, PCR, and serology, fastidious, slow growing bacteria can sometimes be identified in such cases. BCNE accounts for $2.5-31 \%$ of all cases of endocarditis in industrialized countries and for up to $76 \%$ of cases in the developing world.2 Routine blood culture fails to identify a causative organism in $40 \%$ of infectious endocarditis cases at Khon Kaen University Hospital, a tertiary care facility in northeast Thailand, ${ }^{3}$ and the case fatality rate of BCNE in Khon Kaen is $38 \% .^{3}$ A collaborative project aimed at elucidating the causes of BCNE in Khon Kaen was begun in 2010 with objectives to improve clinical outcomes and identify potential preventive interventions. We describe the first known case of Bartonella spp. endocarditis in Thailand.

\section{Case Report}

A 57-year old male poultry farmer with underlying rheumatic heart disease presented to a local hospital with a 5 day history of fever, muscle pain and shortness of breath. He was found to be in congestive heart failure and was transferred to the regional cardiac referral center, Srinagarind Hospital, Khon Kaen, Thailand. On admission, he had fever of $39.0^{\circ} \mathrm{C}$, heart murmurs consistent with aortic stenosis, aortic regurgitation, mitral stenosis and mitral regurgitation, signs of congestive heart failure, anemia and digital clubbing. A transthoracic echocardiogram showed a large, mobile vegetation on the aortic valve (Figure 1) and on the mitral valve.

Intravenous ampicillin and gentamycin were begun on admission and blood transfusions and dopamine circulatory support was given. However, symptoms of congestive heart failure worsened and 8 days after admission the patient underwent aortic and mitral valve replacement. Histopathological examination of the heart valve tissue showed chronic, active, suppurative endocarditis with hemosiderosis.

Three sets of routine aerobic blood cultures were negative. Serological testing was performed using an indirect immunofluourescent antibody assay using antigens prepared from four Bartonella species. Serology was diagnostic for $B$. henselae, with a serum antibody titer of 1:512 at enrollment and a titer of 1:256 twenty-eight days later. $B$. henselae was also demonstrated in heart valve tissue by shell vial culture, by real-time PCR, and by immunohistochemical staining (Figure 2). ${ }^{4}$

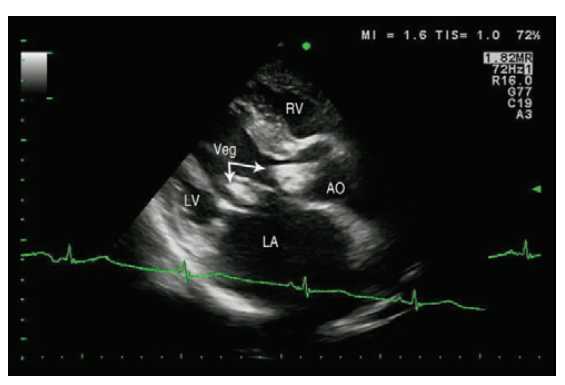

Figure 1. Transthoracic echocardiography showing two large vegetations (Veg), one at the aortic valve (right arrow) and one at the mitral valve (bottom arrow).
Correspondence: George Watt, DDC7 Building 3rd floor, Ministry of Public Health, soi 4, Tivanon Rd., Muang, Nonthaburi 11000 Thailand. Tel. +66.89.927.9928 - Fax: +662.580.0911. E-mail: georgew@th.cdc.gov

Key words: bartonella, endocarditis, cat scratch disease, Thailand.

Acknowledgements: we thank the following investigators for their invaluable help with this study. From Khon Kaen University: Viraphong Lulitanond, Piroon Muksikapan and Burapha Bussadhamma. From the Division of Vector Borne Infectious Diseases, Centers for Disease Control and Prevention, Fort Collins, Colorado: Jennifer K Iverson and M Diaz. From the National Center for Zoonotic, Vector-Borne, and Enteric Diseases, CDC, Atlanta: Christopher Paddock and Patricia Greer in Atlanta. From IEIP, Nonthaburi, Thailand: Sumalee Boonmar and Somsak Thamthitiwat. From The University of the Mediterranean, Marseille, France: PierreEdouard Fournier and Didier Raoult.

Conflict of interest: the authors report no conflicts of interest.

Received for publication: 31 March 2011.

Revision received: 27 May 2011.

Accepted for publication: 27 May 2011.

This work is licensed under a Creative Commons Attribution NonCommercial 3.0 License (CC BYNC 3.0).

(C) Copyright O. Pachirat et al., 2011

Licensee PAGEPress, Italy

Infectious Disease Reports 2011; 3:e9

doi:10.4081/idr.2011.e9

The patient was started on therapy for Bartonella endocarditis with ampicillin and gentamycin. Two months after surgery his cardiac disease stabilized and he was discharged from hospital. However, approximately one

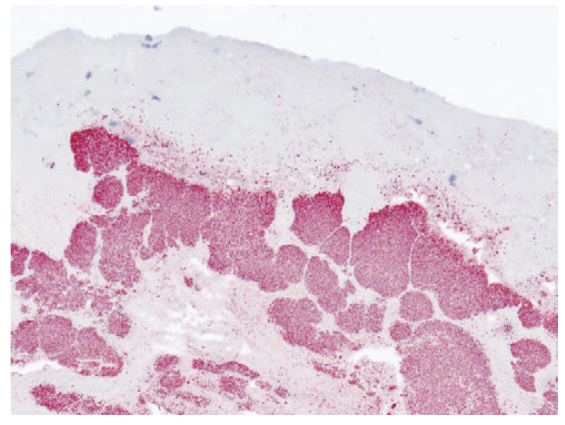

Figure 2. Immunoalkaline phosphatase staining of resected heart valve tissue using a monoclonal antibody reactive only with Bartonella benselae shows bacteria staining red (20X magnification). 
month after discharge, the patient died suddenly at home, apparently from complications of anticoagulation therapy.

\section{Discussion}

Bartonella are fastidious, gram-negative pathogenic bacteria associated with diverse mammalian species. Previous collaborative research conducted by the US CDC's International Emerging Infections Program and Division of Vector Borne Diseases confirmed Bartonella infections among patients with acute undifferentiated fever in Thailand and identified a new species of Bartonella. ${ }^{5-7}$ Bartonella causes a wide spectrum of clinical infections, ranging in severity from asymptomatic or mild febrile illness to endocarditis. There is growing evidence from Europe ${ }^{8}$ the USA9 and from developing countries ${ }^{10}$ that Bartonella are an important cause of human endocarditis. We describe the first reported case of Bartonella spp. endocarditis in Thailand. Our patient was infected with $B$. henselae, the etiologic agent of cat scratch disease. Ownership of cats and underlying heart valve damage are predisposing factors for $B$. henselae endocarditis. ${ }^{11}$ The patient had prior significant aortic and mitral valve defects. Our patient had no cats at home, but his neighbor had a cat from which $B$. henselae was isolated. However, given the high prevalence of
Bartonella infections in cats, we cannot confirm a link to this case. The clinical and public health implications of this finding are important due to the historically high rates of rheumatic heart disease in Thailand, which can often be complicated by infective endocarditis. Increased awareness of this pathogen as a potential agent for infective endocarditis can sensitize physicians to considering Bartonella in the differential diagnosis, to develop diagnostic capacity in Thailand, and help to assure appropriate therapy, which differs from standard therapy for infective endocarditis. Regional surveillance efforts to examine domestic animals, rodents and ectoparasites for Bartonella species are also currently being conducted to help define the epidemiology, clinical spectrum, vectors, and animal reservoirs for Bartonella endocarditis in Thailand.

\section{References}

1. Brouqui P, Raoult D. Endocarditis due to rare and fastidious bacteria. Clin Microbiol Rev 2001;14:177-207.

2. Benslimani A, Fenollar F, Lepidi H, Raoult D. Bacterial zoonoses and infective endocarditis, Algeria. Emerg Infect Dis 2005;11: 216-24.

3. Pachirat 0, Chetchostisakd P, Klungboonkrong V, et al. Infective endocarditis: prevalence, characteristics and mortality in Khon Kaen, 1990-1999. J Med Assoc Thai 2002;85:1-10.

4. Daybell D, Paddock CD, Zaki SR, et al. Disseminated infection with Bartonella henselae as a cause of spontaneous splenic rupture. Clin Infect Dis 2004;39: e:21-4.

5. Kosoy M, Bai Y, Sheff K et al. Identification of Bartonella infections in febrile human patients from Thailand and their potential animal reservoirs. Am J Trop Med Hyg 2010;82:1140-5.

6. Bhengsri $\mathrm{S}$, Bagget $\mathrm{H}$, Peruski L, et al. Bartonella spp. infections, Thailand. Emerg Inf Dis 2010;16:743-5.

7. Kosoy M, Morway C, Sheff KW et al. Bartonella tamiae sp. nov., a newly recognized pathogen isolated from three human patients from Thailand. J Clin Microbiol 2008;46:772-5.

8. Houpikian P, Raoult D. Blood culture-negative endocarditis in a reference center. Medicine 2005;84:162-73.

9. Tunkel AR, Kaye D. Endocarditis with negative blood cultures. N Engl J Med 1992; 326:1215-7.

10. Balakrishnan N, Menon T, Fournier P, Raoult D. Bartonella quintana and Coxiella burnetti as causes of endocarditis, India. Emerg Infec Dis 2008;14:1168-9.

11. Mosbacher ME, Klotz S, Klotz J, Pinnas JL. Bartonella henselae and the potential for arthropod vector-borne transmission. Vector Borne Zoonotic Dis 2011;11:471-7. 